\title{
Application of BP neural network algorithm in bank customer hierarchy
}

\section{system}

\author{
Wang Guowei ${ }^{1,2 a}$, Bai Yunting ${ }^{1,2, b}$, Sun $\mathrm{Yu}^{1,2, c}$ \\ ${ }^{1}$ School of Information Technology, Jilin Agricultural University, Changchun 130118,China; \\ a41422306@qq.com; b307418577@qq.com;‘398223842@qq.com
}

Keywords: Customer hierarchy system,BP neural network,Data mining technology

Abstract: The ever fierce competition in the financial market has highlighted the importance of maintaining good customer relationship for banks. The Customer Relationship Management (CRM) is built on a layered system and the traditional BP neural network has low speed and accuracy. The paper is an attempt to optimize customer layered system by exploring algorithm in BP neural network to arrive at a more precise customer classification system. Momentum-adaptive learning rate adjustment algorithm is used to improve BP neural network algorithm. Since learning speed will decline with more learning, global search is adopted at high learning speed and as it slows down, local search can yield more accurate results. It not only ensures the speed of each epoch but also pinpoints the optimal. The conclusion shows that improved BP neural network algorithm has enhanced accuracy and efficiency of customer segmentation and formed a valid basis for targeted marketing by commercial banks.

\section{Introduction}

City commercial banks have been seen as an emerging force in creating business opportunities around China. The domestic bank customer demand for value-added financial services and assets increasing; secondly, the development of science and technology of information technology to make the original "product centric" banks gradually transferred to the "customer-centric" mode, "customer" has become the focus of competition in the banking sector. Therefore, the CRM is the general trend of banking business development ${ }^{[1-4]}$.

The traditional classification method has been unable to adapt to the diversity of customer data, data mining and knowledge discovery of new technology to extract from the data as the technology, the BP neural network algorithm through the input and output data set, according to the principle of error back-propagation, the changes of the network weights and biases are calculated, gradually approaching the target, provides a new way for the commercial bank customer segmentation ${ }^{[5]}$.

The paper puts forward the momentum method to improve BP neural network algorithm, discovers a better solution and adopts adaptive learning rate method to shorten the training time in $\mathrm{BP}^{[6]}$. By combining the above two methods, the momentum adaptive learning rate adjustment algorithm is obtained. Finally, through the comparison of the test results, the results show that the optimized BP neural network algorithm improves the accuracy and efficiency of customer segmentation.

\section{Bank customer classification principle}

Commercial banks have a large number of customer transaction data, we according to certain classification standard and method, the similar characteristics of the customers are divided into a 
class $^{[7]}$. The principle of customer layering is shown in Figure 1

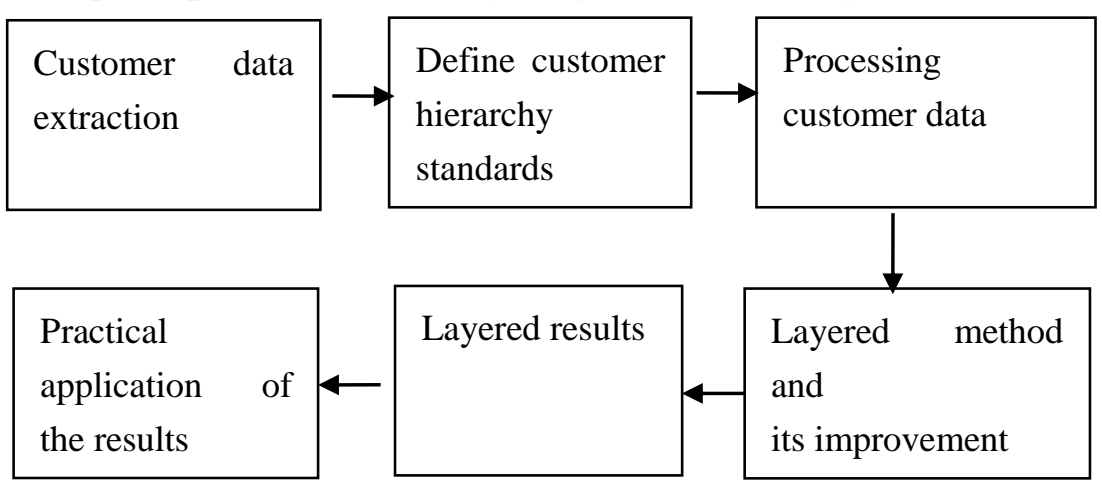

Fig 1.The basic principle of customer hierarchy

BP neural network was proposed by Rinehart and McClelland in 1986. The BP network can learn and store a large number of mapping relations between input and output patterns without revealing the mathematical equations describing such mappings. Its learning rule is to use the steepest descent method to adjust the weights and thresholds of the network by back propagation, so that the sum of squared error of the network is minimum ${ }^{[8-10]}$. Therefore, BP is suitable for modern commercial banks nervous dig out valuable information from the massive historical transaction of customer information.

\section{research methods}

The BP algorithm consists of forward propagation of data flow and backward propagation of error signals. Through the two processes alternately, perform error function gradient descent strategy in weighting vector space, dynamic iterative search a set of weight vectors, so the network error function reaches the minimum value, thus completing the information extraction and memory [11].

Enter a learning sample, which is used $x^{1}, x^{2} \ldots x^{p}, \ldots x^{P}$ to represent. After the ${ }^{p}$ sample was entered into the network, it was lost $y_{j}^{p}(\mathrm{j}=1,2, \ldots \mathrm{M})$. The square error function $E_{p}$ is used to obtain the error of the ${ }^{p}$ sample:

$$
E_{P}=\frac{1}{2} \sum_{j-1}^{m}\left(t_{j}^{p}-y_{j}^{p}\right)^{2}
$$

$t_{j}^{y}$ is the expected output.

The cumulative error BP algorithm is used to adjust the ${ }^{w_{j k}}$, so that the global error $E$ becomes smaller, the global error is reduced

$$
\Delta w_{j k}=-\eta \frac{\partial E}{\partial w_{j k}}=-\eta \frac{\partial}{\partial w_{j k}}\left(\sum_{p-1}^{p} E_{P}\right)=\sum_{p-1}^{p}\left(-\eta \frac{\partial E_{p}}{\partial w_{j k}}\right)
$$

Medium rate: $\eta$ - learning rate

The weights of each neuron in the output layer are adjusted as follows: 


$$
\Delta w_{j k}=\sum_{p-1}^{p} \sum_{j-1}^{m} \eta\left(t_{j}^{p}-y_{j}^{p}\right) f_{2}^{\prime}\left(S_{j}\right) z_{k}
$$

The formula of the weight adjustment of the neurons in the hidden layer is obtained:

$$
\Delta v_{k j}=\sum_{p-1}^{p} \sum_{j-1}^{m} \eta\left(t_{j}^{p}-y_{j}^{p}\right) f_{2}^{\prime}\left(S_{j}\right) w_{j k} f_{1}^{\prime}\left(S_{k}\right) x_{i}
$$

(4)

\section{Improvement of BP algorithm}

In practical application, the BP algorithm is difficult to be competent. When the momentum method is used, the BP neural algorithm can find a better solution; when the adaptive learning rate method is used, the BP algorithm can shorten the training time ${ }^{[12-13]}$. By combining the above two methods, the momentum adaptive learning rate adjustment algorithm is obtained.

\section{Improved BP algorithm with momentum method}

In essence, it is a static gradient descent optimization algorithm., in the amendment of $W(k)$.The specific method of momentum right value adjustment algorithm is to add a part of the last weight adjustment amount to the weight adjustment amount calculated according to the error, as the actual weight adjustment amount, namely:

$$
\Delta W(n)=-\eta \nabla E(n)+\alpha \Delta W(n-1)
$$

In the formula: $\alpha$ is the momentum coefficient, usually $0<\alpha<0.9 ; \quad \eta_{\text {is }}$ the learning rate, the range is between 0.001 and 10 .

\section{adaptive learning rate}

An important reason for the slow convergence rate of standard BP algorithm is that the learning rate is not properly chosen, .The learning rate can be adjusted by the adaptive method shown in Fig. 2.

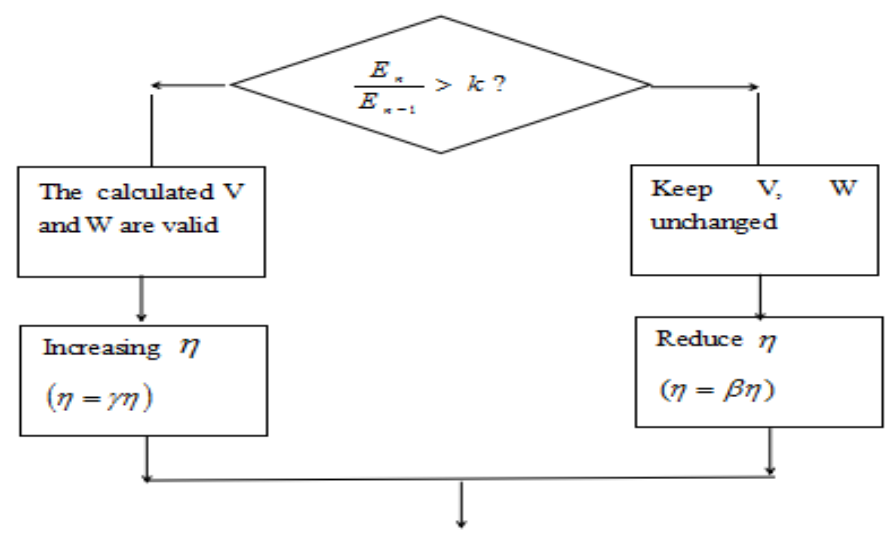

Fig 2.Adaptive learning rate adjustment diagram 
The formula of weight adjustment with additional momentum factor is:

$$
\begin{aligned}
& \Delta w_{i}(k+1)=\left[(1-m c) \eta \delta_{i} p_{j}+m c \Delta w_{i j}(k)\right] \times 0.998^{k} \\
& \Delta v_{i}(k+1)=\left[(1-m c) \eta \delta_{i}+m c \Delta v_{i}(k)\right] \times 0.998^{k}
\end{aligned}
$$

Among them, $\mathrm{k}$ is the number of training; $\mathrm{mc}$ is momentum factor, the general value is about 0.95. The essence of the additional momentum method is to transfer the influence of the last weight change through a momentum factor.

\section{Using improved BP neural network to classify customers in commercial banks}

The basic steps are as follows:

1) extract customer data from commercial banks

2) integrate the original data, delete redundant items, and hide the data involved in privacy

3) using the transaction data, the customers are divided into four categories: high net worth customers, high quality customers, potential customers and ordinary customers.

4) classify the data by BP neural network algorithm, and get the prediction results, and calculate the global error value.

5) using the optimized BP algorithm to predict and calculate the global error.

6) test the customer classification system of commercial banks and output the classification results.

\section{implementation and Application}

\section{simulation environment and data}

Python neural network toolbox is used in processing experiment data to verify the improvement the momentum-adaptive learning rate adjustment algorithm has made to BP neural network algorithm.

Customer data from a commercial bank from 600 customers a year deposit and loan account transactions, transaction details due to the huge amount of data, we use the following methods to integrate data, calculate each customer's comprehensive contribution, make the data more simplified, for the next step of the research.

\section{Error comparison of BP neural network algorithm before and after optimization}

Comparative experiment for the improved algorithm, the experimental results are shown in Figure 3, compared to the same conditions, the number of iterations increasing, former algorithm has slow convergence rate and value in a certain range of output error, falling into local optimal value, the improved error less than before improvement, convergence speed is fast, and the error has been reduced, so as to find the global optimum. 


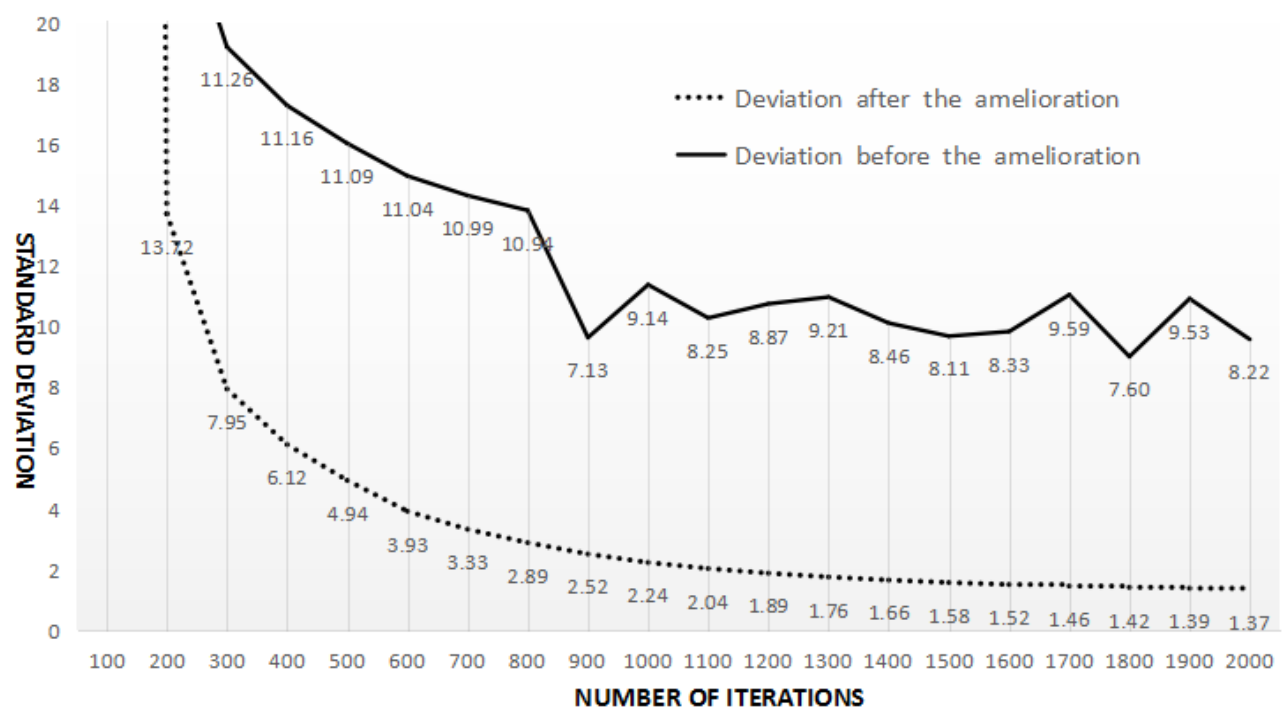

Fig 3. Comparison chart of operation error results

\section{commercial banks for customer segmentation accuracy comparison}

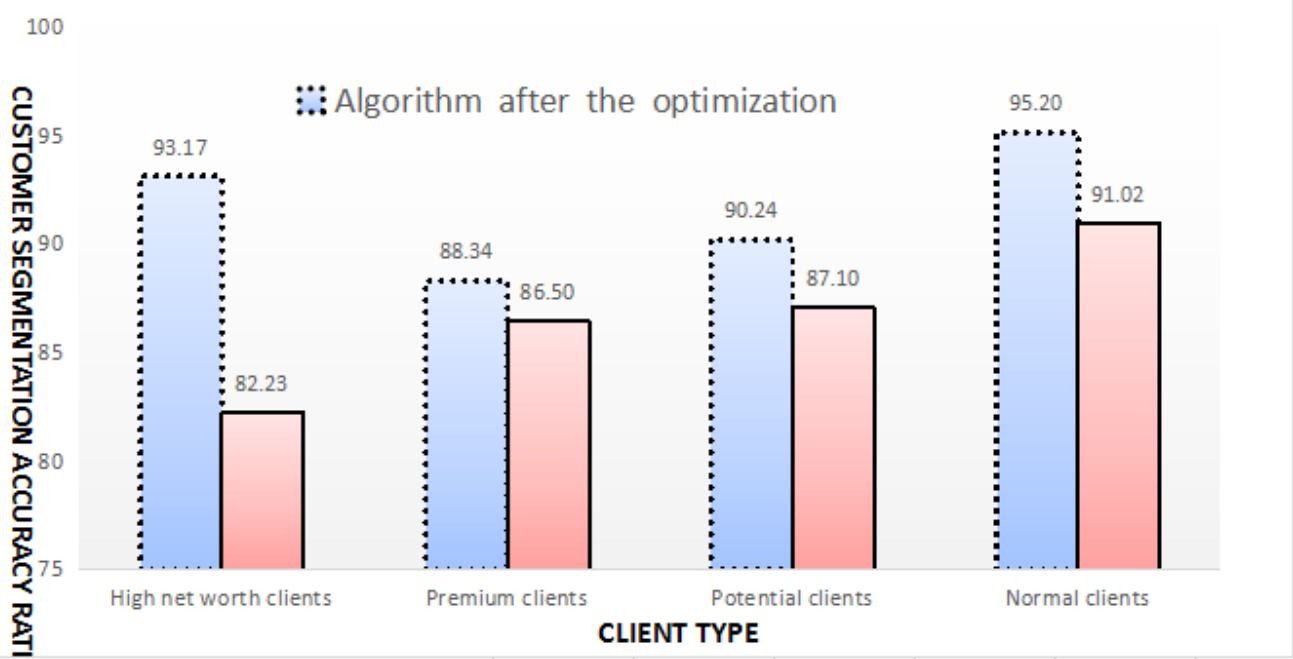

Fig 4.BP algorithm commercial bank customer classification accuracy comparison chart

From Figure 4, the accuracy rate of the improved BP neural network algorithm of customer segmentation is higher than that of the traditional BP algorithm, the main use of additional momentum method to find the global solution; using adaptive learning rate method to shorten the training time.

\section{Conclusion and discussion}

The modern commercial bank system based on CRM advanced concept of "customer centered", to improve the comprehensive competitiveness plays a crucial role; data mining technology and UI analysis of its powerful function in data analysis plays an important role, the combination will 
become the trend of research. Based on the BP neural network algorithm in data mining, this paper proposes to use momentum adaptive learning rate adjustment algorithm to improve the level of commercial bank customers. The results show that the improved algorithm of hierarchical bank customers to improve the accuracy of hierarchical, more efficient classification results, commercial banks can adjust the marketing strategy and service strategy based on hierarchical optimization results, the bank's allocation of resources, so as to improve the profitability of banks.

\section{Acknowledgements}

This work is supported by Jilin Provincial Modern Agriculture Development project -demonstration and application of traceability system of quinoa products based on Internet of things and 3S Technology.

\section{Reference}

[1] Zhang Guozheng. Customer relationship management of data mining in customer classification research based on J] [. Business research, 2006 (13): 153 -155

[2] Yang Weiming. Research on customer relationship management of CCB Zhongshan branch: [Master's Degree Thesis]. Chengdu: University of Electronic Science and technology of China, 2010

[3] Qu Xiao Ning. The application of [J]. simulation in the commercial bank customer classification in $\mathrm{K}$ mean clustering algorithm 2011, 28 (6): 357-360.

[4] Ji described the data mining algorithm. The vocative case study and application of [J]. technology in wind bank CRM, 2011 (5): 26-27.

[5] Liu Kun. Research on student hierarchical algorithm based on BP neural network [J]. science and technology information, 2013 (15): 13-13.

[6] Wang Meiling, Wang Nianping, Li Xiao. Improvement and application of BP neural network algorithm [J]. computer engineering and applications, 2009, 45 (35): 47-48.

[7] Ye Xueying, Li Xiaowei. Customer management and service in modern commercial banks [J]. Journal of Wuhan Agricultural Bank of China Training Institute, 2000 (2): 51-54.

[8] Hu Haifeng, Zhao Kai. Research on data mining technology based on BP neural network [J]. Journal of Pingdingshan University, 2008, 23 (2): 112-114.

[9]Gupta A K, Greenwood G W. Static task allocation using (+ lambda) evolutionary strategies[J]. Information Sciences, 1996, 94 (1-4): 141-150.

[10]WANG Liang, ZHANG Hongwei, YUE Lin, Application of PSO-BP Model. In Short-Term Prediction of Urban Water ConsumptionPSO-BP model with theory and practice, the application of [J]. system in water engineering short-term prediction in the city 2007, 27 (9): 165-170.

[11] Tang Wanmei. BP neural network structure optimization problem [J]. systems engineering theory and practice, 2005, 25 (10): 95-100.

[12] Sun Yu Doherty [D]. the East China Jiaotong University. Power amplifier design improved BP algorithm with adaptive learning rate momentum based on the 2015 .

[13] wave field. Face recognition method of BP neural network based on [J].. Journal of Tongren University, 2017, 19 (3): 43-45. 